\title{
Guest Editorial: Special Issue on Analog, Mixed-Signal, RF, and MEMS Testing
}

\author{
Hsiu-Ming (Sherman) Chang • David C. Keezer
}

Received: 20 August 2012 /Accepted: 20 August 2012 / Published online: 12 September 2012

(C) Springer Science+Business Media, LLC 2012

Test cost has gradually become a major portion of the overall product cost for System-on-Chips (SoCs). Developing efficient methods to test and debug analog, mixed-signal, and radiofrequency (RF) circuitries is of particular interest due to their lengthy test times and increased complexity. In the past, mixedsignal/RF circuits were built as stand-alone chips, so test developments were geared toward characterizing individual building blocks. With increased integration, the controllability and observability to these mixed-signal/RF circuits has become limited. As a result, built-in self-test (BIST) and design-fortestability (DfT) modifications that can help reduce test time and facilitate silicon debug have gained more attention.

This special issue collects 16 state-of-the-art solutions for testing various mixed-signal, RF, and MEMS circuits. The first four papers propose BIST techniques for on-chip mixed-signal, RF, and MEMS modules. In paper 1, Zhang et al. develop an amplitude detector that transforms highfrequency signals into DC/low-frequency components. They then infer high-frequency circuit performance from detectors' output and show that both the system- and component-level performances can be tested with great accuracy. In paper 2, Hung and Hong propose a BIST scheme for ADCs where the control signals are wirelessly transmitted from an HOY tester. They show that the BIST

Responsible Editor: H.-M. Chang

H.-M. S. Chang $(\bowtie)$

Logic Technology Development Division, Intel Corporation, 2501 NW 229th Street, RA3-353,

Hillsboro, OR 97124, USA

e-mail: hsiu-ming.chang@intel.com

\section{C. Keezer}

School of Electrical and Computer Engineering,

Georgia Institute of Technology,

777 Atlantic Drive,

Atlanta, GA 30332-0250, USA

e-mail: david.keezer@ece.gatech.edu test results align well with those produced by conventional methods. In paper 3, Kim and Abraham propose a BIST scheme for testing the setup and hold time of the memory interface. They use a static mode test method to determine pass/fail on the timing specifications, as well as a dynamic mode test method to measure the amount of timing mismatch. In paper 4, Sarraf et al. develop an adaptive technique based on pseudo-random sequences for testing and calibrating MEMS-based accelerometers and gyroscopes.

Papers 5 to 7 focus on techniques for generating accurate and low-cost test signals. In paper 5, Duan et al. incorporate the "stimulus error identification and removal" (SEIR) algorithm into their BIST solution so that they can test high-resolution ADCs without using high precision stimuli, thereby saving cost. For applications that require time or frequency domain test stimulus, in paper 6, Tsai et al. develop a time/frequency-domain signal generator using a sigma-delta modulator, a digital-to-frequency or a digital-totime converter, and a phase locked-loop. Testing ADCs usually requires high-precision signal generation. In paper 7, Wakabayashi et al. propose an algorithm for generating low distortion sine-wave signals using low-resolution (hence, low cost) waveform generators.

Papers 8 to 12 enhance observability of internal analog signals. In paper 8 , Kulovic and Margala introduce a technique for on-chip voltage measurement. On-chip voltages are first converted to the time domain, and subsequently, to the frequency domain using a centralized time-to-digital converter. The distributed manner of voltage-to-time conversion enables concurrent voltage measurement. In paper 9, Maltabas et al. propose a current sensor that is suitable for IDDQ measurement. In paper 10, Gómez et al. exploit the relationship between device transconductance and static power dissipation to observe process variations. In paper 11, Dasnurkar and Abraham propose a built-in current sensor that is suitable for measuring internal bias currents. 
In paper 12, Huang et al. propose a self-test and selfcalibrating scheme for embedded SAR ADCs by characterizing the bit weights of the capacitor array.

In addition to BIST techniques, cost-effective techniques for testing and characterizing mixed-signal circuits are also addressed. In paper 13, Naing et al. present a test method that enables parallel test of up to eight RF devices using a single tester. In paper 14 , Wu et al. describe the use of a single high frequency sine-wave for testing ADC jitter. In paper 15, Long et al. investigate a near-optimal feature vector selection method in the least-square support vector machine (LS-SVM) for analog circuit diagnosis. In paper 16 , Sindia et al. utilize the recent advances in polynomial coefficient and V-transform coefficients to test and diagnose analog circuits.

We enjoy the breadth and the depth of the paper collected in this special issue. We hope you find them interesting and useful. 